\title{
Consequences of stabilising selection for polygenic variation
}

\section{Kenneth Mather}

Department of Genetics, University of Birmingham, P.O. Box 363, Birmingham B15 2TT, U.K.

A number of earlier authors have investigated the consequences for fitness of gene differences affecting the expression of a primary character where stabilising selection is acting in favour of an optimal expression. Assuming that the mean expression must be at or close to the optimum, deviations from the mean have been taken as measures of the deviation from the optimum, and the conclusion reached that, random drift apart, stabilising selection must ultimately lead to fixation of the commoner allele. It is now shown that this approach is incorrect: in an illustrative example the mean cannot be taken as synonymous with the optimum except in the trivial case where they have precisely the same value. Where the mean departs from the optimum, even by sampling variation only, continuing selection for it is effectively self-propagating and directional away from the optimum. Deviation from the optimum itself must be used in investigating the consequences of stabilising selection.

The model used here is based on the biometrical description of the effects of a gene difference on a quantitative character as used by Mather and Jinks (1982). This includes a parameter, $h$, which allows dominance of any magnitude in either direction to be taken into account, and is adapted for the present purpose by introducing an additional parameter, $m$, measuring the departure from the optimum of the mid-point, or mid-parent, which is used biometrically as the origin for measurement of the gene effects. Assuming random mating and the absence of epistasis in the effects on the primary character, it is shown that stabilising selection acting on a pair of alleles (A and a) can have any one of three possible outcomes depending on the relative values of the $\boldsymbol{m}$ and $\boldsymbol{h}$ which characterise the effects of the gene difference on the primary character, viz: (i) a stable equilibrium in the population where in respect of the primary character Aa is nearer to the optimum than both homozygotes; (ii) fixation of the fitter allele where Aa is intermediate between the homozygotes in its departure from the optimum; (iii) a theoretical unstable equilibrium leading to fixation of the commoner allele, where Aa departs further from the optimum than both homozygotes. Only outcome (i) can lead to the conservation of variation in the population.

Preliminary consideration is also given to the interaction in effect on fitness of two gene pairs affecting the same character and segregating simultaneously in the population.

Published data from two experiments on the properties of the conserved variation for two chaeta characters in the Texas population of Drosophila melanogaster, as revealed by half-diallel sets of crosses among 11 and 16 inbred lines respectively, are shown to agree with theoretical expectations derived from the present analysis. Some consequences of differences of overall magnitude in the effects of the mutations by which the variation originated are discussed.

\section{INTRODUCTION}

Stabilising selection is generally accepted as the most prevalent type of natural selection, but there has been no similar consensus about the consequences of this type of selection for the genetic structure of random mating populations. Two views have emerged. Using analytical approaches, Wright (1935), Robertson (1956), Bulmer (1971) and Kimura $(1981,1983)$ have concluded that, random drift apart, continuing stabilising selection will lead to fixation of the more common of two alleles. Kimura (1981) also concludes that under stabilising selection alleles can behave as if negatively overdominant and that then they have a higher chance of fixation by random drift than do unconditionally deleterious mutants with comparable selection coefficients. On the other hand Kojima (1959b), having first (1959a) derived analytically the conditions (expressed in terms of components of genotypic variance) for stable equilibrium where two or more loci are involved, applies these to the situation where selection value depends on the deviation of the expression of a 
character from an optimum value, and so obtains the relevant condition for stable equilibria. He illustrates the application of these criteria by numerical examples using two loci with the same effects and concludes that stable equilibria are possible, given partial or overdominance on the primary scale of expression of the character. Kojima's findings were followed up using similar numerical methods by Lewontin (1964), Jain and Allard (1965) and Singh and Lewontin (1966) and were extended to cover 3 loci, inbreeding and linkage. Again using similar methods but a somewhat different model, Gale and Kearsey (1968) and Kearsey and Gale (1968) also considered the effects of linkage and showed that stable equilibria were possible under stabilising selection, in their case with systems of 2 and 3 linked loci having effects of different magnitude on the character, though not all combinations of linkage disequilibrium and differences of magnitude effects would lead to them.

It is thus of interest to examine further the effects of stabilising selection on allele frequencies and to compare, as far as is possible, the theoretical expectations with such relevant experimental evidence as is available. The consequences of linkage will not, however, be considered. It will be assumed that the population is random mating, that on its primary scale of phenotypic expression the character shows polygenic (quantitative) variation, that the genes governing the variation recombine freely and that they show no epistasis, though they may show dominance.

\section{THEORETICAL CONSIDERATIONS}

\section{Single locus}

With polygenic variation it is expected that as a result of earlier directional selection the mean expression of a phenotypic character contributing significantly to fitness will be at or close to the optimal expression. It might then be expected that selection for the mean expression will be equivalent to stabilising selection for the optimal phenotype, and indeed Wright (1935), Robertson (1956), Bulmer (1971) and Kimura (1981; 1983) all use deviations from the mean as their basis for finding the relative fitnesses of the three genotypes, $\mathrm{AA}, \mathrm{Aa}$ and aa, at a diallelic locus under stabilising selection. Selection for the mean is not, however, equivalent to selection for the optimum as a simple example will show.

Consider two alleles segregating at random in a population. Following Mather and Jinks (1982), the allele tending to increase the expression of the character is designated $\mathrm{A}$ and the reducing allele a. Dominance is taken to be absent and the gene frequencies, $u$ of $\mathrm{A}$, and $v(=1-u)$ of a, to be equal. Let the mid-point $(M)$ between the average expressions of AA and aa be at the optimum $(\mathrm{O})$, with AA deviating from it by $d$ and aa by $-d$. This mid-point is the average expression of $\mathrm{Aa}$ and, since $u=v=\frac{1}{2}$, is also the mean expression of the character in the population. With fitness falling off symmetrically on the two sides of the optimum cum mean the frequencies of the two alleles are at equilibrium.

Suppose, however, that as a result of random drift the frequency of $A$ in the next generation increases to $\frac{1}{2}+\Delta u_{1}$, that of a being correspondingly reduced. The mean is no longer at the midpoint (which may be taken as 0 ) but has risen to $2 \Delta u_{1}$, even though the optimum is still the same. As the earlier authors have shown, selection for this new mean will result in $u$ rising by a further increment, which itself will be proportional to $\Delta u_{1}$, to give $u=\frac{1}{2}+\Delta u_{1}+\Delta u_{2}$. The mean will also rise correspondingly to $2\left(\Delta u_{1}+\Delta u_{2}\right)$ and further generations of selection will continue the trend of increase in both $u$ and the mean. The selection will thus be chasing an ever-receding mean until ultimately $u=1$ and allele $\mathrm{A}$ is fixed in the population. This selection has indeed been building up an effect of random drift, but it has not been stabilising selection. Rather it must be regarded as a self-propagating form of directional selection whose effects are similar to a steady trend change in the environment.

When however we treat the mid-point as marking the optimum no matter how far the mean changes, a different picture emerges. The three genotypes will retain the same selective values no matter how the gene frequencies change; Aa, lying at the optimum, will have the greatest fitness, with AA and aa having the fitness of phenotypes whose expressions of the character lie at $d$ and $-d$ respectively from the optimum. So, given that $d^{2}$ is small by comparison with the variance, $\sigma^{2}$, of the character's expression in the population, both homozygotes will have fitnesses less than that of $\mathrm{Aa}$ by an amount proportional to $d^{2}$. This is the classical situation leading to stable polymorphism. In our case the reduction of fitness is the same for AA and aa, so $s_{\mathrm{A}}=s_{\mathrm{a}}$. In passing from one generation to the next $u$ will alter by $\Delta u=\left(u v d^{2} / \sigma^{2} \bar{w}\right)$ $\left(v s_{\mathrm{a}}-u s_{\mathrm{A}}\right)$ where $\bar{w}$ is the mean fitness of the population, and since $\Delta u$ is opposite in sign to $u-\hat{u}$, where $\hat{u}$ is the frequency at equilibrium, $u$ will move towards $\hat{u}$, and subject to random drift the equilibrium will be stable at $\hat{u}=\hat{v}$. 
In our example, by contrast with continuing selection for the mean expression, selection for the optimal expression is stabilising: subject to sampling variation, it keeps the mean at the optimum, it counteracts sampling variation rather than facilitating it, and it conserves polymorphism in respect of alleles $A$ and a. Nor is this surprising. The optimal phenotype is that which best enables the individual to cope with the demands made and the pressures imposed on it by the environment. It is determined from the outside and is the property of the individual. The mean expression of the character, on the other hand, is determined by the frequencies of the alleles and their combinations in the population. It affords an estimate of the optimal phenotype only insofar as directional selection has moved it to the optimum and stabilising selection is keeping it there.

The example that we have been discussing is over-simple in that it assumes that dominance is absent and that the mid-point coincides with the optimum. Neither assumption is valid in general. Dominance is a common feature of the gene pairs contributing to polygenic variation and so far as we know may be of any magnitude within the range between complete for the increasing allele and complete for the decreasing allele. (It should be noted that the use of $\mathrm{A}$ and $\mathrm{a}$ for denoting the increasing and decreasing alleles respectively car- ries no implication in respect of dominance). Equally the position of the mid-point in relation to the optimal expression of the character must depend on the direction and size of the effect on the expression of the character of the mutation which gave rise to the allelic difference: only in the unlikely event of the mutation being in the right direction and of just the right size will the mid-point be at the optimum or, also taking dominance into account, the average phenotypic expression of the heterozygote be at the optimum. We must therefore allow in our analysis for both dominance and departure of $M$ the mid-point from $\mathrm{O}$, the optimum. We thus need two further parameters, $h$ as a measure of the departure of the phenotype of Aa from the mid-point, and $m$ as a measure of the departure of the mid-point from the optimum (fig. 1). Both $m$ and $h$ take sign, and are conveniently measured taking $d$ as the unit. $d$ itself is expressed in units of $\sigma$, the standard deviation of the frequency distribution, assumed to be normal, followed by $x$, the expression of the primary character, $x$ being measured from $x_{0}$ the optimal expression. $d$, and with it $m$ and $h$, are assumed to be sufficiently small not to cause significant departure from normality of the sub-distributions which they determine. As already noted, random mating and the absence of both linkage disequilibrium and epistasis in respect of the

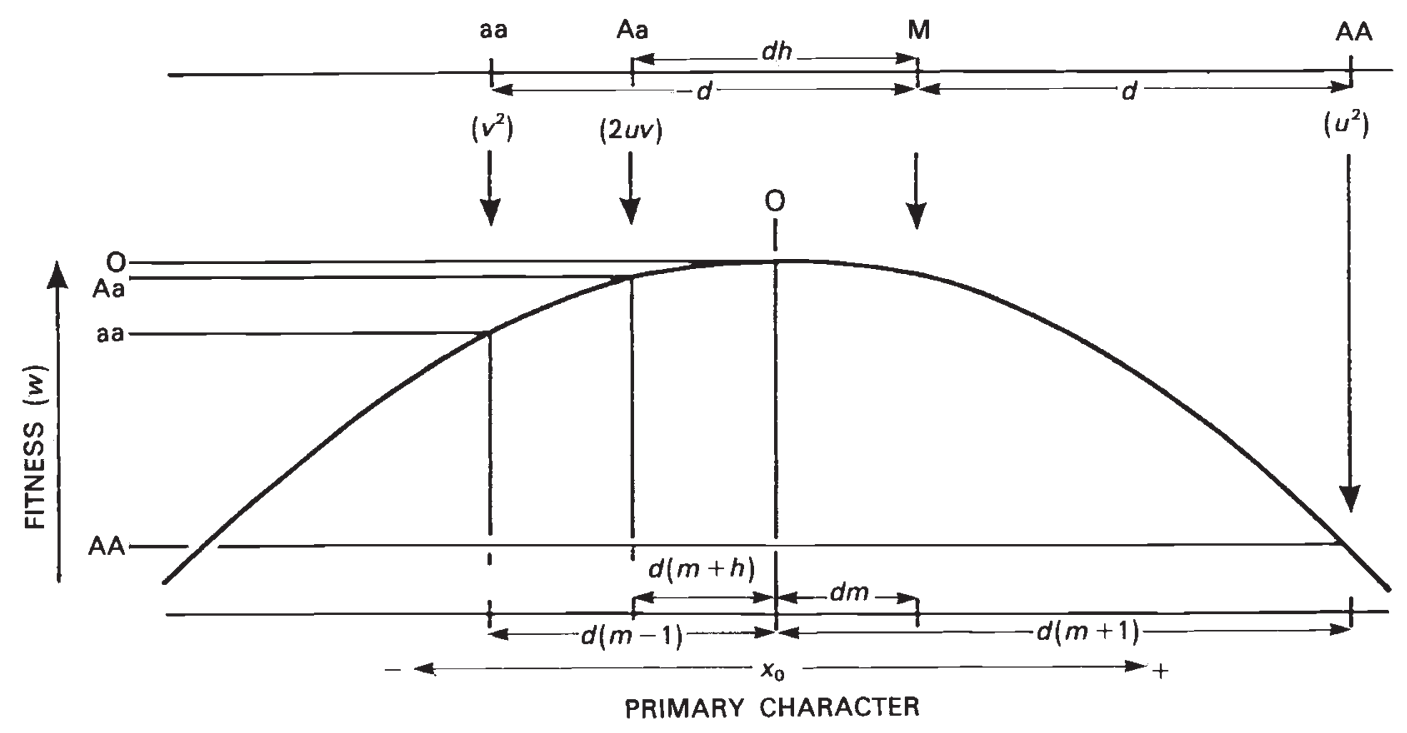

Figure 1 Above: the use of the parameters $d$ and $h$ to represent the differences in expression of the primary character in biometrical terms. The mid-point (M) between the homozygotes is taken as the origin. $h$ may take sign, being negative in the case illustrated. The frequencies of the three genotypes under random mating are also shown. Below: the adaptation of this system for the analysis of stabilising selection. The optimum phenotype $(0)$ is now taken as the origin and a third parameter $(m)$ is introduced to denote the departure of $\mathrm{M}$ from $\mathrm{O}$. $m$ can also take sign. 
expression of the primary character are also assumed. The population mean, $\bar{x}$, of the character will be $d_{\mathrm{a}}\left[m_{\mathrm{a}}+\left(u_{\mathrm{a}}-\mathrm{v}_{\mathrm{a}}\right)+2 u_{\mathrm{a}} v_{\mathrm{a}} h_{\mathrm{a}}\right]$ summed over all the $n$ loci contributing to the variation.

Following Kimura (1981) we define the relative fitnesses of AA, Aa and aa as:

$$
\begin{aligned}
w_{i j} & =\int_{-\infty}^{\infty} w(x) f\left(x_{0}-x_{i j}\right) d x \\
& =b_{0}-b_{1} x_{i j}+b_{2} x_{i j}^{2} / 2
\end{aligned}
$$

where $i$ and $j$ each can be $\mathrm{A}$ or a and $x_{i j}$ is the departure of the average phenotype of the relevant A,a from the optimum, corresponding to Kimura's $\mathrm{a}_{i j}$ which is the departure from the mean;

$$
b_{0}=\int_{-\infty}^{\infty} w(x) f(x) d x
$$

with $f(x)$ replaced by its first derivative to give $b_{1}$ and by its second derivative to give $b_{2}$.

We assume, with Kimura, that both $f(x)$ and $w(x)$ follow normal distributions, $f(x)=$ $(1 / \sqrt{2 \pi}) \exp \left[x^{2} / 2\right]$ and $w(x)=\exp \left[-k x^{2}\right]$. Then $b_{1} / b_{0}=\bar{x} \lambda$ and $b_{2} / b_{0}=\bar{x}^{2} \lambda^{2}-\lambda$, where $\lambda=$ $2 k /(1+2 k)$. Since following a period of selection towards a stable optimum we expect $x$ not to depart significantly from the optimal expression we assume, again with Kimura, that the mean is at the optimum, i.e., $\bar{x}=0$ since we have taken the optimum as the origin for $x$. Then $b_{1} / b_{0}=0$ and $b_{2} / b_{0}=-\lambda$.

Taking the values of $x_{i j}$ as shown in fig. 1 , and substituting these values for $b_{1} / b_{0}$ and $b_{2} / b_{0}$ in equation (1) we obtain:

$$
\begin{gathered}
w_{\mathrm{AA}} / b_{0}=1-\lambda d^{2}(m+1)^{2} / 2 \\
w_{\mathrm{Aa}} / b_{0}=1-\lambda d^{2}(m+h)^{2} / 2 \\
w_{\mathrm{aa}} / b_{0}=1-\lambda d^{2}(m-1)^{2} / 2
\end{gathered}
$$

giving

$$
\begin{aligned}
w_{\mathrm{A}} / b_{0}= & 1-\lambda d^{2}\left[u(m+1)^{2}+v(m+h)^{2}\right] / 2 \\
w_{\mathrm{a}} / b_{0}= & 1-\lambda d^{2}\left[v(m-1)^{2}+u(m+h)^{2}\right] / 2 \\
\bar{w} / b_{0}= & 1-\lambda d^{2}\left[u^{2}(m+1)^{2}\right. \\
& \left.+2 u v(m+h)^{2}+v^{2}(m-1)^{2}\right] / 2
\end{aligned}
$$

with

$$
\begin{aligned}
\Delta u= & u\left(w_{\mathrm{A}}-\bar{w}\right) / \bar{w}=\frac{\lambda}{2 \bar{w}} u v d^{2} \\
& \times\left[(v-u)\left(1-h^{2}-2 m h\right)-2 m\right] .
\end{aligned}
$$

Apart from $\lambda / 2 \bar{w}$ this equation can also be derived, more directly, using Robertson's (1956) approach.

\section{Setting}

$\Delta u=\frac{\lambda}{2 \bar{w}} \hat{u} \hat{v} d^{2}\left[(\hat{v}-\hat{u})\left(1-h^{2}-2 m h\right)-2 m\right]=0$.

where $\hat{u}$ and $\hat{v}$ denote the gene frequencies at equilibrium, and leaving aside the special cases of $u, v$, or $d=0$, equilibrium is thus achieved when

$$
\hat{v}-\hat{u}=2 m /\left(1-h^{2}-2 m h\right) \text {. }
$$

If we write $\hat{u}+e$ for $u$ and $\hat{v}-e$ for $v$ in equation (2), and subtract (3) from it, we obtain $(\lambda / 2 \bar{w}) u v d^{2}\left[-2 e\left(1-h^{2}-2 m h\right)\right]=\Delta u$. Thus where $h^{2}+2 m h<1, u-\hat{u}$ and $\Delta u$ are of opposite sign and the equilibrium is stable. But if $h^{2}+2 m h>$ $1, u-\hat{u}$ and $\Delta u$ are of the same sign and the equilibrium is unstable. Also, the closer $1-h^{2}-$ $2 m h$ approaches to 0 , the smaller $\Delta u$ becomes, and the effect of sampling variation relative to selection pressure increases correspondingly.

Taking first our simple example where the midpoint is at the optimum $(m=0)$ and dominance is absent $(h=0)$, both homozygotes will be at a disadvantage, $d^{2}$, relative to the heterozygote which will be at the optimum. Thus $\Delta u$ will always equal $(\lambda / 2 \bar{w}) u v d^{2}(-2 e)$ and the equilibrium will always be stable at $\hat{u}=\hat{v}=\frac{1}{2}$. But if $h \neq 0, \Delta u=$ $(\lambda / 2 \bar{w}) u v d^{2}\left[-2 e\left(1-h^{2}\right)\right]$ and although there will be a stable equilibrium at $\hat{u}=\hat{v}$ so long as $|h|<1$, the selective pressure will diminish as dominance increases in either direction. At $h= \pm 1$ selection pressure vanishes as $\Delta u$ becomes 0 . As Kojima pointed out there will be no equilibrium when dominance is complete. Random sampling will determine the gene frequencies and if fixation occurs it may be of either allele. With over-dominance in either direction, Aa will be less fit than both AA and aa, and instability supervenes, with the consequence that the more frequent allele, whichever it may be, will be favoured by selection. At the same time, however, this is a situation in which, as Kimura has shown, the chance of an initially rare allele achieving fixation by random drift is enhanced by the "negative over-dominance" which the heterozygote is showing.

The situation is more complex when the midpoint is not at the optimum $(m \neq 0)$. From equation (4), when $1-h^{2}-2 m h>0$ and $m$ is positive $\hat{v}-\hat{u}=$ 1 at $h=-1$ or $1-2 m$, and $x_{\mathrm{Aa}}$, the departure of Aa from the optimum expressed in units of $d$, is then $m+h=m-1$ or $1-m$, which are equally spaced on opposite sides of the optimum. Furthermore $x_{\mathrm{aa}}=m-1$ is the departure of aa from the optimum when aa is the fitter homozygote. Allele a will clearly move to fixation. When $m$ is negative AA is the fitter homozygote and $\hat{v}-\hat{u}=-1, x_{\mathrm{Aa}}=$ 
$m+h$ is again either $1-m$ or $m-1$ : Aa will coincide in fitness with the fitter homozygote, and allele A will move to fixation. In both cases intermediate values of $h$ will give intermediate values of $m+h$ and the departure of Aa from the optimum on the primary scale will be less than that of even the fitter homozygote. It is easy to show that equilibria will then arise and will be stable because $\Delta u$ and $\boldsymbol{u}-\hat{u}$ are of opposite sign.

These relations are illustrated in fig. 2 for the cases where both $m$ and $h$ lie within the range 1 to -1 . It will be seen that, as might be expected, the cases where $m$ is positive and negative are symmetrical in the sense that each is transposed into the other by rotation of the figure through $180^{\circ}$. Stable equilibrium is achieved over a wider range of values of $h$ the smaller $m$ becomes and it is also commoner when $h$ is of opposite sign to $m$ : indeed where $|m|>\frac{1}{2}$ it is achieved only when $h$ and $m$ are of opposite sign. Thus $(\hat{v}-\hat{u}) h$ must be negative in the majority of cases. The importance of this relation will become clearer when we turn to consider the experimental evidence later.

Similar ranges of stable equilibria are obtained when $|m|>1$ and $|h|$ is both $>1$ and of opposite sign to $m$. Nevertheless these ranges still lie between $h=-1$ and $1-2 m$ for positive $m$ and between $h=1$ or $-(1+2 m)$ for negative $m$, giving $m+h$ intermediate between $1-m$ and $m-1$, with Aa thus departing less from the optimum than even the fitter homozygote. So stable equilibria can be obtained for single loci with over-dominance, as well as with partial dominance on the primary scale, just as Kojima found with his special cases of two loci.

Equilibria between the frequencies of the two alleles can arise only when $\hat{v}-\hat{u}=$ $2 m /(1-h-2 m h)$ lies between 1 and -1 , which requires that $2 m<1-h^{2}-2 m h$. When $2 m>$ $1-h^{2}-2 m h, \hat{v}-\hat{u}$ becomes either $>1$ or $<-1$ according to the sign of $m$. This is genetically impossible and the allele giving the fitter homozygote will become fixed just as it does when $\hat{v}-\hat{u}=1$ or -1 .

$1-h^{2}-2 m h$ will change sign when $h^{2}+2 m h$ moves from being $<1$ to being $>1$. This change takes place when $1-h^{2}-2 m h=0$, which requires that $h=-m \pm \sqrt{m^{2}+1}$, which gives $x_{\mathrm{Aa}}=m+h=$ $\pm \sqrt{m^{2}+1}$. These two values are again equally spaced on opposite sides of the optimum, and apart from the case where $m=0$ they lie outside the values of $m+h$ at which the fixation of the fitter allele replaces stable equilibria. When $h^{2}+2 m h-$ $1>0$ but $<2 m, \hat{v}-\hat{u}$ still takes a genetically impossible value and fixation of the fitter allele will still take place. The situation changes, however, when $h^{2}+2 m h-1$ becomes $>2 m$. This transition takes place at $2 m /\left(h^{2}+2 m h-1\right)=1$, which arises when $h=-(1+2 m)$ or 1 , and $x_{\mathrm{Aa}}=$ $m+h= \pm(m+1)$. These are the departures from the optimum not only of Aa but also of the less fit of the two homozygotes, $x_{\mathrm{aa}}$ being $-(m+1)$

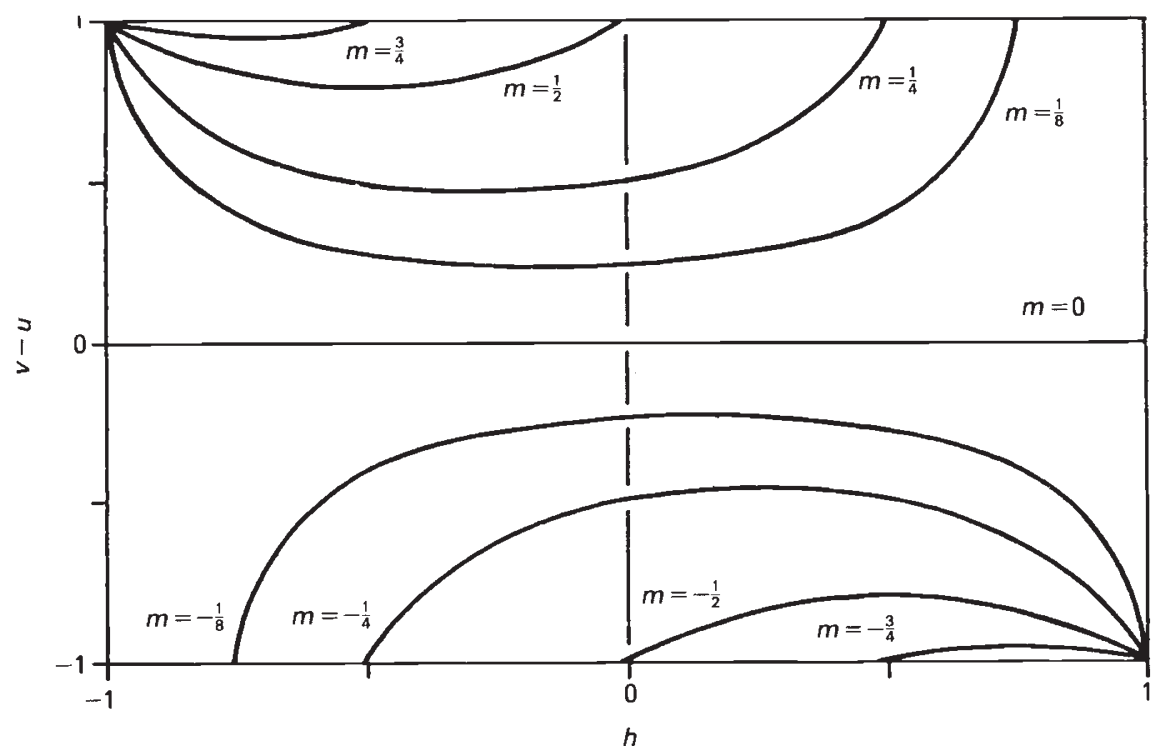

Figure 2 The relation of the equilibrium value of $v-u$ (ordinate) to $h$ (abscissa) for nine selected values of $m$, covering the range -1 to 1 for both $h$ and $m$. 
when aa is the less fit and $x_{\mathrm{AA}}=m+1$ when AA is. When $h^{2}+2 m h-1>2 m$ equilibrium values can theoretically be found for $v-u$; but, as we have already seen, $\Delta u$ and $u-\hat{u}$ now have the same sign: any equilibrium will be unstable and will generally lead to fixation of the commoner allele. This again is the situation discussed by Kimura (1981).

We can thus recognise three possible outcomes of stabilising selection. When $x_{\mathrm{Aa}}$ lies between $m-1$ and $1-m, A a$ is fitter than both homozygotes and stable equilibria are expected to arise; when $x_{\mathrm{Aa}}$ lies between $m-1$ and $-(m+1)$ or between $1-m$ and $1+m \mathrm{Aa}$ is intermediate in fitness between the homozygotes and the allele giving the fitter one is expected to go to fixation; and when $x_{\mathrm{Aa}}$ is $<-(m+1)$ or $>m+1$, Aa is less fit than both homozygotes and unstable equilibria can theoretically arise, though generally this will lead to fixation of the commoner allele. In all three cases, the expected outcome is, of course, subject to modification if random drift is great enough to have a significant effect. We may finally note that, no matter what the value of $h$ may be, the second outcome cannot occur when $m=0$ : phenotypically the two homozygotes will depart equally from the optimum, albeit in opposite direction, and hence will show no difference in fitness.

\section{Two loci}

Although we are assuming no epistasis of genes at different loci in their effects on the primary scale of the expression of the character, we must be prepared to find that they can show interaction in their effects on the relative fitnesses of the individuals. Where two gene pairs, $\mathrm{A}-\mathrm{a}$ and $\mathrm{B}-\mathrm{b}$, are segregating with free recombination in a population the value of the mean expression of the character for each of the nine genotypes in the absence of epistasis will be the sum of the two corresponding values for loci $\mathrm{A}, \mathrm{a}$ and $\mathrm{B}, \mathrm{b}$; and their frequencies in the population are assumed to be the products of the frequencies of the relevant $\mathrm{A}, \mathrm{a}$ and $\mathrm{B}, \mathrm{b}$ genotypes. Thus the equivalent of $x_{i j}^{2}$ in the case of a single locus will be $\left[d_{\mathrm{a}}\left(m_{\mathrm{a}}+1\right)+\right.$ $\left.d_{\mathrm{b}}\left(m_{\mathrm{b}}+1\right)\right]^{2}$ for AABB, whose frequency is taken to be $u_{\mathrm{a}}^{2} u_{b}^{2}$. This can conveniently be rewritten as $d_{\mathrm{a}}^{2}\left(m_{\mathrm{a}}+1\right)^{2}+2 d_{\mathrm{a}} d_{\mathrm{b}}\left(m_{\mathrm{a}}+1\right) d_{\mathrm{b}}\left(m_{\mathrm{b}}+1\right)+d_{\mathrm{b}}^{2}\left(m_{\mathrm{b}}+1\right)^{2}$.

Following our earlier procedure we can obtain:

$$
\begin{aligned}
2_{\mathrm{BB}} \bar{w}_{\mathrm{a}} \Delta u_{\mathrm{a}}= & \lambda u_{\mathrm{a}} v_{\mathrm{a}}\left\{d_{\mathrm{a}}^{2}\right. \\
& \times\left[\left(v_{\mathrm{a}}+u_{\mathrm{a}}\right)\left(1-h_{\mathrm{a}}^{2}-2 h_{\mathrm{a}} m_{\mathrm{a}}\right)-2 m_{\mathrm{a}}\right] \\
& \left.\left.-2 d_{\mathrm{a}}\left[1+\left(u_{\mathrm{a}}-v_{\mathrm{a}}\right) h_{\mathrm{a}}\right] d_{\mathrm{b}}\left(m_{\mathrm{b}}+1\right)\right]\right\}
\end{aligned}
$$

in the presence of $\mathrm{BB}$. In the presence of $\mathrm{Bb}$ we obtain a similar expression but with ${ }_{\mathrm{Bb}} \bar{w}_{\mathrm{a}}$ and $\left(m_{\mathrm{b}}+\right.$ $\left.h_{\mathrm{b}}\right)$ in place of ${ }_{\mathrm{BB}} \bar{w}_{\mathrm{a}}$ and $\left(m_{\mathrm{b}}+1\right)$, and in the presence of bb again a similar expression but with ${ }_{\mathrm{bb}} \bar{w}_{\mathrm{a}}$ and $\left(m_{\mathrm{b}}-1\right)$. Averaging over the three genotypes for $\mathrm{Bb}$, according to their frequencies and dividing through by $\bar{w}_{\mathrm{a}}=u_{\mathrm{b} \mathrm{BB}}^{2} w_{\mathrm{a}}+2 u_{\mathrm{b}} v_{\mathrm{b} \mathrm{Bb}} \bar{w}_{\mathrm{a}}+u_{\mathrm{b} \mathrm{bb}}^{2} \bar{w}_{\mathrm{a}}$ gives

$$
\begin{aligned}
\Delta u_{\mathrm{a}}= & \frac{\lambda u_{\mathrm{a}} v_{\mathrm{a}}}{2 \bar{w}_{\mathrm{a}}}\left\{d _ { \mathrm { a } } ^ { 2 } \left[\left(v_{\mathrm{a}}-u_{\mathrm{a}}\right)\right.\right. \\
& \left.\times\left(1-h_{\mathrm{a}}^{2}-2 h_{\mathrm{a}} m_{\mathrm{a}}\right)-2 m_{\mathrm{a}}\right] \\
& -2 d_{\mathrm{a}}\left[1+\left(u_{\mathrm{a}}-v_{\mathrm{a}}\right) h_{\mathrm{a}}\right] d_{\mathrm{b}} \\
& \left.\times\left[m_{\mathrm{b}}+u_{\mathrm{b}}-v_{\mathrm{b}}+2 u_{\mathrm{b}} v_{\mathrm{b}} h_{\mathrm{b}}\right)\right\} .
\end{aligned}
$$

The first part of this expression is that already obtained in equation (2) for the single locus case. The second part reflects the effect of interaction with $\mathrm{B}-\mathrm{b}$ on the reaction of $\mathrm{A}$, a to stabilising selection. It will be noted that this interaction vanishes when the mean effect of B-b on the expression of the character is 0 . Assuming that higher order interactions are small enough to be negligible and summing over all relevant loci this condition becomes $\mathrm{S}_{\mathrm{b}}^{n}\left(m_{\mathrm{b}}+u_{\mathrm{b}}-v_{\mathrm{b}}+2 u_{\mathrm{b}} v_{\mathrm{b}} h_{\mathrm{b}}\right)=0$, which is virtually the same as the condition, assumed in the single locus analysis, that the mean expression of the character is at the optimum: i.e., $\bar{x}=0$.

This expectation takes no account of linkage. It may be disturbed also by the quasi-linkage, or gametic disequilibrium, that can arise from epistasis in the determination of fitness, even in the absence of true linkage (Moran, 1964; Kimura, 1965), though such a disturbance may be small. This remains a matter for future investigation.

\section{EXPERIMENTAL EVIDENCE}

A number of experiments have been reported in which "stabilising" selection was applied for various characters in Drosophila (references in Mather, 1983), but none of these is apposite to our present enquiry as the selection was for the mean value of the chaeta number and the results were related to changes in the variance, especially the additive genetic variance, of the population. Kearsey and Barnes (1970) have, however, shown that the fitnesses of individuals from the Texas cage population, maintained in this Department, are related to their numbers of sternopleural chaetae. Their capacity for survival under relatively crowded conditions is at a maximum where, as 
adults, their chaeta numbers are near the mean, and it declines as the chaeta number departs increasingly from this central value no matter in which direction. Linney et al. (1971) further showed that the decline could not be related to the level of heterozygosity of the flies, as the same differences in capacity for survival were observed when the individuals of differing chaeta numbers came from inbred lines. In other words sternopleural chaeta number is displaying the effects of natural stabilising selection under laboratory condition.

The history of the Texas cage population is given by Caligari and Mather (1980). In brief, the population was started with the progeny of 30 inseminated females caught in Texas in late 1965 and has been maintained as a cage population since early 1966. In 1967 some 100 inbreeding lines were initiated from it by single pair matings, and were maintained by single pair sib-matings. Most of the lines died out quickly, but 18 were still in existence some 10 years later. These provided the material for the analysis of the genetical architecture of sternopleural chaeta number to which we now refer.

Caligari and Mather (l.c.) discussed the question of whether, in the light of their observations, these 18 inbreds constitute a fair sample of the population and concluded that there was no observational reason for doubting that they do so, provided that we accept that in both the population and the inbreds the dominant alleles are preponderantly the common alleles for the relevant genes. They took their analysis further by a half-diallel experiment using 11 of the inbreds as parents. In this they followed both the mean number of sternopleural chaetae averaged over flies reared at $18^{\circ}$ and $25^{\circ} \mathrm{C}$ (character $\mathrm{M}$ ), and the sensitivity of the chaeta number to the temperature difference (character S). Caligari (1981) carried out a similar half-diallel experiment using, however, 16 of the inbred lines as parents, but raising the flies only at $25^{\circ} \mathrm{C}$. In addition to sternopleural chaeta number he also recorded the numbers of coxal chaeta on each of the front $(\mathrm{F})$, middle $(\mathrm{M})$ and rear $(\mathrm{R})$ pairs of legs. The results relevant to our present discussion have been extracted from the two papers and are set out in table 1 . All the variances and covariances had already been corrected for nonheritable effects by the original authors.

The results for the coxal chaetae are not presented in relation to the three different pairs of legs, $\mathrm{F}, \mathrm{M}$ and $\mathrm{R}$, but in terms of the three classes of genes, $\alpha, \beta$ and $\gamma$, which Mather and Hanks (1978) postulated to account for the differences in chaeta number between them. $\alpha$ genes affect the chaeta numbers of all three pairs of legs; $\beta$ genes affect those of the $M$ and $F$ legs but not the R; and $\gamma$ genes affect only those of the $\mathrm{F}$ legs. Assuming these three classes of gene, predictions can be made about the response of the $F, M$ and $R$ chaeta numbers to various forms of directional selection and these expectations were verified by Hanks and Mather (1978): the three classes of gene effectively respond independently to selection. Caligari also reports that he found no covariation between the inbred lines in respect of their numbers of sternopleural chaetae and coxal chaetae on their F legs,

Table 1 Data from the Drosophila half-diallels reported by Caligari and Mather (C and M) and Caligari (C), relating to sternopleural chaeta number $(\mathrm{St})$ and coxal chaeta number $(\mathrm{Co})$. For sternopleural chaetae two characters, mean number $(\mathrm{M})$ and temperature sensitivity (S), were followed and for coxal chaetae the components attributable to the $\alpha, \beta$ and $\gamma$ types of gene. The values of the four components of variation are given in the upper part of the table, and those of $\overline{(v-u) h}$ and its components in the lower part

\begin{tabular}{|c|c|c|c|c|c|c|c|}
\hline & $\mathbf{M}$ & $\begin{array}{l}\text { and } M \text { ) } \\
S\end{array}$ & $\begin{array}{l}\text { St }(C) \\
M\end{array}$ & $\alpha$ & $\begin{array}{l}\mathrm{Co}(\mathrm{C}) \text { V } \\
\beta\end{array}$ & $\gamma$ & \\
\hline$D_{P}$ & $5 \cdot 422$ & $0 \cdot 501$ & $7 \cdot 577$ & 0.973 & 1.546 & $1 \cdot 721$ & \\
\hline$D_{R}$ & $3 \cdot 556$ & $0 \cdot 184$ & $4 \cdot 262$ & 0.500 & $1 \cdot 102$ & 1.405 & \\
\hline$H_{R}$ & 0.624 & $0 \cdot 172$ & 0.678 & $0 \cdot 103$ & 0.032 & $0 \cdot 230$ & \\
\hline$\sqrt{D_{P}, D_{R}}$ & $4 \cdot 391$ & $0 \cdot 304$ & $5 \cdot 683$ & 0.697 & $1 \cdot 305$ & $1 \cdot 555$ & \\
\hline$\overline{(\underline{v}-u) h}$ direct & $-0 \cdot 211$ & -0.349 & -0.282 & $-0 \cdot 320$ & $-0 \cdot 175$ & -0.095 & -0.239 \\
\hline$|\bar{h}|$ & 0.439 & 0.640 & 0.465 & 0.509 & $0 \cdot 290$ & 0.375 & 0.453 \\
\hline$|\overline{v-u}|$ & 0.636 & 0.400 & 0.766 & 0.768 & 0.868 & 0.227 & 0.611 \\
\hline$(v-u) h$ synthetic & -0.279 & -0.256 & -0.356 & -0.390 & -0.252 & -0.085 & $-0 \cdot 270$ \\
\hline
\end{tabular}


so suggesting that the number of sternopleural chaetae would respond to selection independently of the coxals.

The numbers of polymorphic loci making experimentally detectable contributions to the variation in sternopleural chaeta number was estimated to be 16 by Kearsey and Barnes, (l.c.) and Davies (1971) arrived at a closely similar number, 15 , using a different technique and different material. These will be minimal numbers as one must assume that other polymorphic loci were present but making differences too small to be detected; but even with 15-16 loci the number of inbreds used in the experiments under discussion is too small for each locus to be making a fully independent contribution to the variances and covariances calculable from the diallel experiments: even some of the genes borne on different chromosomes may be correlated with one another in their transmission from the inbred parents to the $F_{1}$ 's in the diallel, and this must be taken into account in interpreting the data from the experiments.

The design and analysis of diallel experiments are described in detail by Mather and Jinks (1982). For our present purposes we need only note that estimates of four components of heritable variation are yielded by such experiments:

(1) $D_{P}=\mathrm{S}\left\{4 u v d^{2}\right\}$, where $\mathrm{S}$ indicates summation over all relevant loci, is the genetic component of variation among the parental inbred lines assuming the absence of epistasis;

(2) $D_{R}=\mathrm{S}\left\{4 u v d^{2}\left[1+(v-u) h^{2}\right]\right\}$ is the corresponding $D$ component of variation in the population;

(3) $D_{W}=\mathrm{S}\left\{4 u v d^{2}[1+(v-u) h]\right\}$ is the $D$ component of covariance of parent and offspring from random mating in the population;

(4) $H_{R}=\mathrm{S}\left\{16 u^{2} v^{2} d^{2} h^{2}\right\}$ is the dominance, or $H$, component of variation in the population.

The genetic variance of the population is $\frac{1}{2} D_{R}+$ $\frac{1}{4} H_{R}$ in terms of these components.

The numerical values of the four components observed by Caligari and Mather (1980) and by Caligari (1981) are set out in the upper part of table 1 . In all the cases $D_{P}>D_{W}>D_{R}$, so showing that $(v-u) h$ must be preponderantly negative. This indicates that (a) $(v-u) \neq 0$ in general; (b) $h \neq 0$ in general; (c) $h$ is preponderantly of opposite sign to $(v-u)$; and (d) $m \neq 0$ in general also, since even with dominance present equilibrium is at $v-u=0$ unless $m \neq 0$. These inferences, which are still valid even if the relevant genes are not all independent of each other in transmission from the inbred parents to the $F_{1}$ 's in the diallel, are clearly in accord with expectation from the theoretical consideration in the previous sections.

$D_{w}$ cannot exceed $\sqrt{D_{P} D_{R}}$, whose values are also given in table 1, except by sampling variation. It will equal $\sqrt{D_{P} D_{R}}$ if $(v-u) h$ is constant over all the relevant loci, but sampling error apart, it will fall short of $\sqrt{D_{P} D_{R}}$ where $(v-u) h$ varies. In fact $D_{W}$ exceeds $\sqrt{D_{P} D_{R}}$ only in the case of Caligari and Mather's character $\operatorname{St}(\mathrm{S})$. This must be due to sampling variation and indeed Caligari and Mather note that this character shows a relatively large proportion of non-genetic relative to genetic variation. $D_{W}$ and $\sqrt{D_{P} D_{R}}$ are almost exactly equal for the coxal chaetae $\gamma$ genes. This implies that there is little variation of $(v-u) h$ for these genes, though this inference is not necessarily valid if the genes in question are not wholly independent in their transmission from inbred parents to $F_{1}$ 's.

An estimate of the weighted mean value of $(v-u) h$ can be obtained from $\left(D_{W}-D_{P} / D_{P}\right)=$ $\overline{(v-u) h}$, the weights being $u v d^{2}$. Similar weighted mean estimates of the further quantities relevant to our discussion can be obtained if we find $\left(D_{P}+\right.$ $\left.D_{R}-2 D_{W}-H_{R}\right) / D_{P}=\bar{h}^{2}$ from which the magnitude, though not the sign of $\bar{h}$ can be derived. Also dividing $H_{R}$ by $D_{P}$ gives $4 \bar{u} \bar{v} \bar{h}^{2}$, which in turn yields $u v$ when divided by $4 \bar{h}^{2}$. The values of yields $u v$ when divided by $4 \bar{h}^{2}$. The values of $\overline{(v-u) h}, \bar{h}$ and $\overline{u v}$ so obtained are given in the lower part of table 1 .

the characters observed so showing that, irrespective of any lack of independence in transmission from inbreds to $F_{1}$ 's in the diallel, the sign of $(u-v) h$ must be preponderantly negative, or in other words that the dominant allele must preponderantly be the common allele as Caligari and Mather pointed out and as our present theoretical analysis leads us to expect. This expectation applies irrespective of the direction of dominance, as we already observed in the previous section, and indeed we know from the two half-diallel experiments that dominance is ambidirectional. This in turn reflects the expectation that ambidirectional dominance will be associated with stabilising selection and unidirectional dominance with continuing directional selection (Mather, 1960). This distinction was borne out by observations on a variety of characters of the two kinds in Drosophila (Breese and Mather, 1960; Kearsey and Kojima, 1967).

With ambidirectional dominance the average value of $h$, when measured directly, will reflect the balance of the dominance in the two directions. It may indeed approximate to 0 in $F_{1}$ 's from unselec- 
ted lines and even in $\mathrm{F}_{1}$ 's from two lines selected in opposite directions, so frequently leading to the assumption that dominance is absent. Where, however, as in the present case, $\bar{h}$ is found as $\sqrt{h^{2}}$, sign will play no part in estimating its value. It is of interest therefore that the value of $\bar{h}$ shows a striking consistency, ranging from 0.64 to 0.29 with an average of 0.453 : indeed the two half-diallels yield estimates of 0.439 and 0.465 for dominance in the case of sternopleural chaeta number.

The value of $\overline{u v}$, which cannot be $<0$ or $>0 \cdot 25$, is useful in two ways. In the first place, since $u v$ forms part of the weighting factors, it tells us that where either $u$ or $v$ is small the gene difference in question will make a correspondingly low contribution to the weighted means that we must use in estimating the components of variation and that where $u$ and $v$ are more nearly equal, the gene difference will make a correspondingly large contribution to the weighted mean. We are however interested chiefly in the value of $(v-u) h$. An estimate of the magnitude of $(\overline{v-u})$ can be obtained from $\bar{u} \bar{v}$, though again it tells us nothing about sign. Now as $u v$ tends to its maximum, $0 \cdot 25,(v-u)$ and with it $(v-u) h$ tends to 0 . Also the value of $(v-u)$ is directly related to $m$. So when either $m$ or $h$ (or both) is small $(v-u) h$ will be small, and the major contribution to the estimate of $\overline{(v-u) h}$ will thus depend chiefly on gene differences with intermediate values of $h$ and $m$. The values of $\mid \overrightarrow{v-u}$ so derived are also given in table 1 , and their mean corresponds to the commoner allele being approximately four times as frequent as the rarer one. Inspection of fig. 2 suggests that such an equilibrium would arise when $|m|$ lay preponderantly between 0.25 and 0.5 while $|h|$ lay preponderantly between 0 and 0.5 with $h$ opposite in sign to $m$.

Knowing the values of $|\bar{h}|$ and $|\overline{v-}| \bar{u} \mid$ we can obtain a synthetic estimate of $\overline{(v-u) h}$, though its sign will not be known. The estimates so yielded by the data from the two half-diallel experiments again are given in table 1 . If we assume that, as theoretical considerations lead us to expect, $(v-u)$ and $h$ are preponderantly of opposite sign, all the $\overline{(v-u) h}$ will be negative and can be compared for consistency with the direct estimate of $\overline{(v-u) h}$. The two estimates are not expected to be equal, partly because the first direct estimates drew on information from $D_{W}$ and $D_{P}$ alone, whereas the second draws on $D_{R}$ and $H_{R}$ also; and partly because the product of two means does not in general equal the mean of the individual products. The two estimates should nevertheless be correlated, as indeed they are, with $r=0.772$ and $P$ lying between $0 \cdot 10$ and $0 \cdot 05$, even though based on only six comparisons.

Thus the data from these two experiments display internal consistency when analysed in relation to our theoretical expectation, and they conform with these expectations, despite the experiments not having been designed specifically to test them.

\section{CONCLUSION}

The relation of the value of $v-u$ to those of $m$ and $h$, on which it depends, is complex. Potentially it can have three possible outcomes according to the actual values of $m$ and $h$; (i) the production of a stable polymorphism where $m$ and $h$ are such that the heterozygote deviates less from the optimum, and hence is fitter, than both homozygotes, (ii) the selective fixation of the allele giving the fitter homozygote, where the deviation of the heterozygote from the optimum, and hence its fitness, it intermediate between those of the two homozygotes, (iii) the situation where an unstable equilibrium is theoretically possible because the heterozygote deviates more from the optimum, and hence is less fit, than both homozygotes. This last situation must also generally lead to fixation of one allele, though not necessarily the innately fitter, because the commoner allele has a selective advantage over the rarer by reason of its greater frequency. Basically this is the situation which Kimura has discussed, and in which he recognises the effect of stabilising selection as potentially facilitating fixation by random drift.

Our concern has been chiefly with the production of balanced polymorphisms largely because their occurrence as an outcome of stabilising selection has been denied by earlier writers who assumed that the mean expression of the character was synonymous with the optimal expression, even when at the same time data already existed in the literature that could provide at least a first test of any conclusions that might be reached. No such data exist which would provide corresponding information on changes that lead to fixation. There is thus a need for further experiments of appropriate kinds.

Once it is accepted that the mean expression is at or close to the optimal expression, stable equilibria are expected to arise given certain relations between $m$ and $h$ and the experimental data agree with this expectation, as we have seen. Such polymorphism will conserve genetic variation and indeed under stabilising selection must play a major part in building up and conserving the pool 
of genetic variation that selection experiments have repeatedly shown to be a characteristic feature of wild populations. Transient polymorphisms will of course be generated by mutants which because they are moving towards fixation at the expense of their less fit progenitors, or because of random drift, have yet to attain fixation or elimination. These, however, can hardly be making other than minor contributions to the genetic variance of characters such as abdominal and sternopleural chaeta number in populations in view of the very small increment that mutation has been found to add to the variance in each generation (Clayton and Robertson, 1955; and see reviews by Mukai, 1979, and Mather, 1983).

The chance of a mutation affecting the expression of a character under stabilising selection, leading ultimately to a stable equilibrium and so contributing to the population's store of genetic variation, depends on the values of $m$ and $h$ that characterise its effects. When $m$ lies outside the range -1 to 1 , stable equilibrium becomes possible only if there is an appropriate level of overdominance with $h$ having sign opposite to that of $m$. The evidence from the two experiments that we have described offers no suggestion of overdominance, and we may therefore confine consideration (at least until evidence of overdominance appears) to the situation where $m$ and $h$ both lie between -1 and 1.

As we have seen, the range of values of $h$ that make stable equilibrium possible varies with $m$ (fig. 2). At $m=0$ any value of $h$ between -1 and 1 is effective, but the further $m$ moves from 0 the narrower the range of effective $h$ value, until as $m$ approaches 1 only values of $h$ approaching -1 , and vice versa, can lead to stable equilibrium. If we assume that all possible combinations in the values of $m$ and $h$ are equally likely to characterise the effects of a mutation, only half of them will offer the possibility of a stable equilibrium in twothirds of which $m$ and $h$ will be of opposite sign. The other half of the combinations of $m$ and $h$ would be expected to result sooner or later in the fixation of one or other allele as a result either of steady selection in its favour or to the mutation having given rise to the situation which Kimura has discussed. It might be noted in passing, too, that barring overdominance all mutations giving $m$ lying outside the range -1 to 1 would lead to fixation for one or other of these two reasons. More mutations would therefore lead to either refixation of the original allele or fixation of the mutant than to stable equilibria. Such fixation of mutant alleles could clearly lead to increase in the differences between populations even where these were under the pressure of stabilising selection centred on the same optimum. But fixation could neither enhance nor assist in conserving the pool of genetic variation within a population, upon which must primarily depend adjustment of the character by response to any directional selection that might supervene.

The parameter $d$ plays no direct part in determining whether stabilising selection will favour a stable equilibrium between the alleles or fixation of one of them. Nor does it play any part in determining the value of $v-u$ and hence of $(v-$ $u) h$ at any equilibrium that might be reached. $d^{2}$ appears, however, in the common factor of the expression for $\Delta u$ (equation 3 ), and it will therefore affect the rate of approach to equilibrium and indeed the magnitude of the selective pressure on the alleles. It also appears, again with $u v$, in the common factor of the contribution that the locus in question makes to the additive genetic variation of the population. Since $d$ is a characteristic of the mutation which produces the allelic difference it will not be constant over all the loci which contribute to the variation in expression of the character in question: indeed it may not even be constant for successive mutations at the same locus. Where the mutation has taken place in, for example, a silent sequence, or has given rise to a synonymous codon, it will be marked by a molecular change, but will be expected to have no effect on phenotype or fitness (Kimura, 1985). Under other circumstances, however, where it affects the action of the gene it will produce a change in both phenotype and fitness, greater or lesser according to the function of the sequence in which it has occurred and the nature of the mutation itself. The value of $d$ characterising the mutation will vary accordingly, and with it the magnitude of selection pressure it will encounter and the contribution it can make to the genetic variance of the population.

The bigger $d$ is, the larger this contribution will be and the less it will be subject to the effects of random drift. At the same time, since the variation to which it gives rise in the population is part of the background variance for other gene differences, the larger it is the greater will be the effects of random drift at these other loci. Thus $d$ is also basic to our understanding of the consequences of stabilising selection, though not by determining the outcome of the selection, whether stable equilibrium or fixation, but by affecting both the pressure of selection of the gene pair and the contribution it makes to the genetic variance of the population. 
Acknowledgement I am much indebted to Dr J. S. Gale for his continuing, helpful interest in this investigation.

\section{REFERENCES}

BREESE, E. L. AND MATHER, K. 1960. The organisation of polygenic activity within a chromosome in Drosophila. II. Viability. Heredity, 14, 375-400.

BULMER, M. G. 1971. The stability of equilibria under selection. Heredity, 27, 157-162.

CALIGARI, P. D. S. 1981. The selectively optimal phenotypes of the coxal chaetae in Drosophila melanogaster. Heredity, 47, 79-85.

CALIGARI, P. D. S. AND MATHER, K. 1980. Dominance, allele frequency and selection in a population of Drosophila melanogaster. Proc. Roy. Soc. Lond. B., 208, 163-187.

CLAYTON, G. AND ROBERTSON, A. 1955. Mutation and quantitative variation. Am. Nat., 87, 151-158.

DAVIES, R. W. 1971. The genetic relationship of two quantitative characters in Drosophila melanogaster. II. Location of the effects. Genetics, 69, 363-375.

GALE, J. S. AND KEARSEY, M. J. 1968. Stable equilibria under stabilising selection in the absence of dominance. Heredity, 23, 553-561.

HANKS, M. J. AND MATHER, K. 1978. Genetics of coxal chaetae in Drosophila melanogaster. II. Responses to selection. Proc. Roy. Soc. Lond. B., 202, 211-230.

JAIN, S. K. AND ALLARD, R. W. 1965. The nature and stability of equilibria under optimising selection. Proc. Natl. Acad. Sci. USA, 54, 1436-1443.

KEARSEY, M. J. AND BARNES, B. W. 1970. Variation for metrical characters in Drosophila populations. II. Natural selection. Heredity, 25, 11-21.

KEARSEY, M. J. AND GALE, J. S. 1968. Stabilising selection in the absence of dominance; an additional note. Heredity, $23,617-620$.

KEARSEY, M. J. AND KOJIMA, K. 1967. The genetic architecture of body weight and egg hatchability in Drosophila melanogaster. Genetics, 56, 23-37.

KIMURA, M. 1965. Attainment of quasi-linkage disequilibrium when gene frequencies are changing by natural selection. Genetics, 52, 875-890.
KIMURA, M. 1981. Possibility of extensive neutral evolution under stabilising selection with special reference to nonrandom usage of synonymous codons. Proc. Natl. Acad. Sci. USA, 78, 5773-5777.

KIMURA, M. 1983. The Neutral Theory of Molecular Evolution. Cambridge University Press.

Kimura, M. 1985. DNA and the neutral theory. Phil. Trans. Roy. Soc. Lond. B., 312, 343-354.

KoJiMA, K. 1959a. Role of epistasis and overdominance in stability of equilibria with selection. Proc. Natl. Acad. Sci. USA, 45, 984-989.

KOJIMA, K. $1959 \mathrm{~b}$. Stable equilibria for the optimum model. Proc. Natl. Acad. Sci. USA, 45, 989-993.

LEWONTIN, R. C. 1964. The interaction of selection and linkage. II. Optimum models. Genetics, 50, 757-782.

LINNEY, R., BARNES, B. W. AND KEARSEY, M. J. 1971. Variation for metrical characters in Drosophila populations. III. The nature of selection. Heredity, 27, 163-174.

MATHER, K. 1960. Evolution in polygenic systems. Int. Colloquium on Evolution and Genetics, Rome: Acad. Naz dei Lincei, pp. 131-152.

MATHER, K. 1983. Response to selection. In The Genetics and Biology of Drosophila. 3C Ashburner, M., Carson, H. L. and Thompson, J. N. Jr. (eds.) Academic Press, London. pp. 155-221.

MATHER, K. AND HANKS, M. J. 1978. Genetics of coxal chaetae in Drosophila melanogaster. I. Variation in gene action. Heredity, 40, 71-96.

MATHER, K. AND JINKS, J. L. 1982. Biometrical Genetics (3rd Edn.) Chapman and Hall, London.

MORAN, P. A. P. 1964. On the nonexistence of adaptive topographia. Ann. Hum. Genet. Lond., 27, 383-393.

MUKAI, T. 1979. Polygenic mutation. In Quantitative Genetic Variation, Thompson, J. N. Jr. and Thoday, J. M. (eds.) Academic Press, London, pp. 177-196.

ROBERTSON, A. 1956. The effect of selection against extreme deviants based on deviation or on homozygosis. J. Genet., 54, 236-248.

SINGH, M. AND LEWONTIN, R. C. 1966. Stable equilibria under optimising selection. Proc. Natl. Acad. Sci. USA, 56, 13451348.

WRIGHT, S. 1935. Evolution in populations in approximate equilibria. J. Genet., 30, 257-266. 